GA-A24179

\title{
DISCHARGE IMPROVEMENT THROUGH CONTROL OF NEOCLASSICAL TEARING MODES BY LOCALIZED ECCD IN DIII-D
}

\author{
by \\ R. PRATER, R.J. LA HAYE, J. LOHR, T.C. LUCE, C.C. PETTY, \\ J.R. FERRON, D.A. HUMPHREYS, E.J. STRAIT, F.W. PERKINS, \\ and R.W. HARVEY
}




\section{DISCLAIMER}

This report was prepared as an account of work sponsored by an agency of the United States Government. Neither the United States Government nor any agency thereof, nor any of their employees, makes any warranty, express or implied, or assumes any legal liability or responsibility for the accuracy, completeness, or usefulness of any information, apparatus, product, or process disclosed, or represents that its use would not infringe privately owned rights. Reference herein to any specific commercial product, process, or service by trade name, trademark, manufacturer, or otherwise, does not necessarily constitute or imply its endorsement, recommendation, or favoring by the United States Government or any agency thereof. The views and opinions of authors expressed herein do not necessarily state or reflect those of the United States Government or any agency thereof. 


\title{
DISCHARGE IMPROVEMENT THROUGH CONTROL OF NEOCLASSICAL TEARING MODES BY LOCALIZED ECCD IN DIII-D
}

\author{
by \\ R. PRATER, R.J. LA HAYE, J. LOHR, T.C. LUCE, C.C. PETTY, \\ J.R. FERRON, D.A. HUMPHREYS, E.J. STRAIT, F.W. PERKINS, ${ }^{*}$ \\ and R.W. HARVEY ${ }^{\dagger}$
}

This is a preprint of a paper to be submitted for publication in Nucl. Fusion.

*Princeton Plasma Physics Laboratory, Princeton, New Jersey.

${ }^{\dagger}$ CompX, Del Mar, California.

Work supported by

the U.S. Department of Energy under

Contract Nos. DE-AC03-99ER54463, DE-AC02-76CH03073,

and Grant No. DE-FG03-99ER54541

GENERAL ATOMICS PROJECT 30033

OCTOBER 2002 


\begin{abstract}
Neoclassical tearing modes (NTMs) are MHD modes which can limit the performance of high beta discharges in tokamaks, in some cases leading to a major disruption. The destabilizing effect which results in NTM growth is a helical decrease in the bootstrap current caused by a local reduction of the plasma pressure gradient by "seed" magnetic islands. The NTM is particularly well suited to control since the mode is linearly stable although nonlinearly unstable, so if the island amplitude can be decreased below a threshold size the mode will decay and vanish. One means of shrinking the island is the replacement of the "missing" bootstrap current by a localized current generated by electron cyclotron current drive (ECCD). This method has been applied to the $m=3 / n=2$ neoclassical tearing mode in DIII-D, in H-mode plasmas with ongoing ELMs and sawteeth, both of which generate seed islands periodically. In the case of the $3 / 2$ mode, full suppression was obtained robustly by applying about $1.5 \mathrm{MW}$ of ECCD very near the rational surface of the mode. When the mode first appears in the plasma the stored energy decreases by $20 \%$, but after the mode is stabilized by the ECCD the beta may be raised above the initial threshold pressure by $20 \%$ by additional neutral beam heating, thereby generating an improvement in the limiting beta of nearly a factor 2 . An innovative automated search algorithm was implemented to find and retain the optimum location for the ECCD in the presence of the mode.
\end{abstract}




\section{INTRODUCTION}

Experiments on the DIII-D tokamak have shown that in many cases the plasma performance is limited by the onset of neoclassical tearing modes [1]. Higher order modes like the $m=3 / n=2$ mode (where $\mathrm{m}$ is the poloidal mode number and $\mathrm{n}$ is the toroidal mode number) limit the achievable $\beta$ (=plasma pressure/magnetic pressure) and reduce the energy confinement time. Lower order modes like the $m=2 / n=1$ mode lead to a large loss of plasma pressure and frequently to a disruption. For a reactor these limitations may make the difference between economic success and failure [2]. These observations provide strong motivation for control of these magnetohydrodynamic (MHD) instabilities.

Neoclassical tearing modes (NTMs) are magnetic islands which may grow due to a helically perturbed bootstrap current [3]. The helical loss in bootstrap current is caused by a local reduction of the pressure gradient within the island, since the bootstrap current is generated by radial pressure gradients. It was pointed out several years ago that the NTM may theoretically be stabilized by the application of a local current driven within the magnetic island to mitigate the reduction of the bootstrap current [4,5]. These authors pointed out that electron cyclotron current drive (ECCD) is a particularly promising approach for this purpose, since ECCD may be highly localized near the intersection of the EC wave with a low order cyclotron resonance. Due to the short wavelength (compared to the antenna size and the plasma gradient scale length) of the EC wave, the direction and dispersion of the EC wave bundle may be easily controlled in order to keep the ECCD near the center of the island.

Experiments on the ASDEX-U tokamak first demonstrated the efficacy of this approach [6]. These experiments showed that the amplitude of the $m=3 / n=2$ NTM can be strongly reduced or fully suppressed through the application of ECCD at the optimum radial location. Later experiments $[7,8]$ on DIII-D showed that ECCD can completely stabilize the $3 / 2$ NTM even in the presence of vigorous sawteeth and ELMs, which are both known to effectively trigger NTMs. Similar results were obtained on the JT-60U tokamak [9].

The present work advances the state of the art by showing that suppression of the $3 / 2$ NTM leads to improved stability, so that the plasma pressure may be increased by at least $60 \%$ without further instability. Additionally, several means of maintaining the optimum location of the ECCD in the presence of changes in the plasma have been implemented. Such changes may occur as a shift of the plasma major radius due to the increase in plasma $\beta$ when the mode is stabilized or a shift in the location of the ECCD if the electron temperature or density change. The results validate suppression of NTMs by ECCD as a valid and robust means of improving the performance of tokamaks at high beta. 


\section{MODIFIED RUTHERFORD EQUATION}

The basis of the analysis of the NTM is the well-known modified Rutherford equation [4,5] for the growth of an island with a full width of w. Following work by La Haye [8] and Perkins [10], we write the Rutherford equation in the form

$$
\frac{\tau_{\mathrm{R}}}{\mathrm{r}} \frac{\mathrm{dw}}{\mathrm{dt}}=\Delta^{\prime} \mathrm{r}+\frac{128}{3 \pi} \frac{\mathrm{j}_{\mathrm{BS}}}{\mathrm{j}_{\mathrm{q}}} \frac{\mathrm{r}}{\mathrm{s}} \frac{1}{\mathrm{w}}\left[\left(1-\frac{\mathrm{w}_{\mathrm{pol}}^{2}}{\mathrm{w}^{2}}\right)-K_{1}\left(\mathrm{w} / \delta_{\mathrm{EC}}, \Delta \mathrm{R} / \delta_{\mathrm{EC}}\right) \frac{\mathrm{j}_{\mathrm{EC}}}{\mathrm{j}_{\mathrm{BS}}}\right] .
$$

In this equation $\tau_{R}=\mu_{0} \sigma r^{2} / 1.22$ is the resistive skin time, $r$ is the minor radius, $\Delta^{\prime}$ is the classical stability parameter, $\mathrm{jBS}_{\mathrm{BS}}$ is the bootstrap current density, $\mathrm{j}_{\mathrm{q}}=2 \mathrm{~B}_{\mathrm{t}} / \mu_{0} \mathrm{Rq}$ is a quantity characteristic of the equilibrium current density such that $\mathrm{j}_{\mathrm{q}}$ provides a constant safety factor $\mathrm{q}$ for a tokamak, and $\mathrm{r} / \mathrm{s}=\mathrm{q} /(\mathrm{dq} / \mathrm{dr})$. The first term on the right hand side of Eq. (1) is the classical growth term, with $\Delta^{\prime}$ negative in these experiments; otherwise, the classical tearing mode would grow. The second term becomes positive (tending to growth) when w is sufficiently large, w > $w_{\text {pol }}$. Here, $w_{\text {pol }}$ introduces stabilizing effects which become significant at small island size, like the polarization current due to the diamagnetic drift, finite perpendicular transport, and finite Larmor radius or drift orbit effects. The last term in Eq. (1) represents the effects of ECCD. The ratio $\mathrm{jEC}_{\mathrm{E}} \mathrm{j}_{\mathrm{BS}}$ is the figure of merit, where $\mathrm{jEC}_{\mathrm{EC}}$ is the local peak current density generated by the ECCD. Typically, this ratio must be of at least order unity for the ECCD to have a significant effect on the instability. The function $K_{1}$, the effectiveness of the ECCD, takes into account the effects of displacement $\triangle \mathrm{R}$ of the minor radius of the ECCD from the radius of the island and of the relative widths of the ECCD and the magnetic island. Here, $\delta_{\mathrm{EC}}$ is the full width at half maximum of the radial profile of the ECCD, which is calculated to be close to Gaussian in shape. The function $\mathrm{K}_{1}$ has been numerically evaluated by Perkins and Harvey [11] and a spline interpolation to these evaluations is used in this paper.

Equation (1) may be plotted for parameters typical of the DIII-D discharges used in the $m=3 / n=2$ NTM experiments which will be described later in this paper, as shown in Fig. 1 . In experiments the $3 / 2$ island has a saturation size of about $7.5 \mathrm{~cm}$. This is determined from measurements of $T_{e}$ fluctuations by electron cyclotron emission (ECE). The value $\Delta^{\prime} r=-3.2$ is fit to yield this saturated island width. Without ECCD the threshold size for growth is around $2 \mathrm{~cm}$, and the growth rate is the top curve in Fig. 1(a). If ECCD is applied with perfect alignment and with sufficient power that $\mathrm{jEC}_{\mathrm{EC}}=0.5 \mathrm{jBS}$, then the second curve of Fig. 1(a) is obtained. The saturated island size is strongly reduced, from $7.5 \mathrm{~cm}$ to $3.5 \mathrm{~cm}$. If the EC power is increased so that $\mathrm{jEC}_{\mathrm{E}}=\mathrm{jBS}$, then the third curve of Fig. 1(a) is obtained. In this case, the growth rate is negative for any island size and the initially saturated mode amplitude will decrease slowly to about $3 \mathrm{~cm}$ and then increasingly rapidly shrink to zero. 

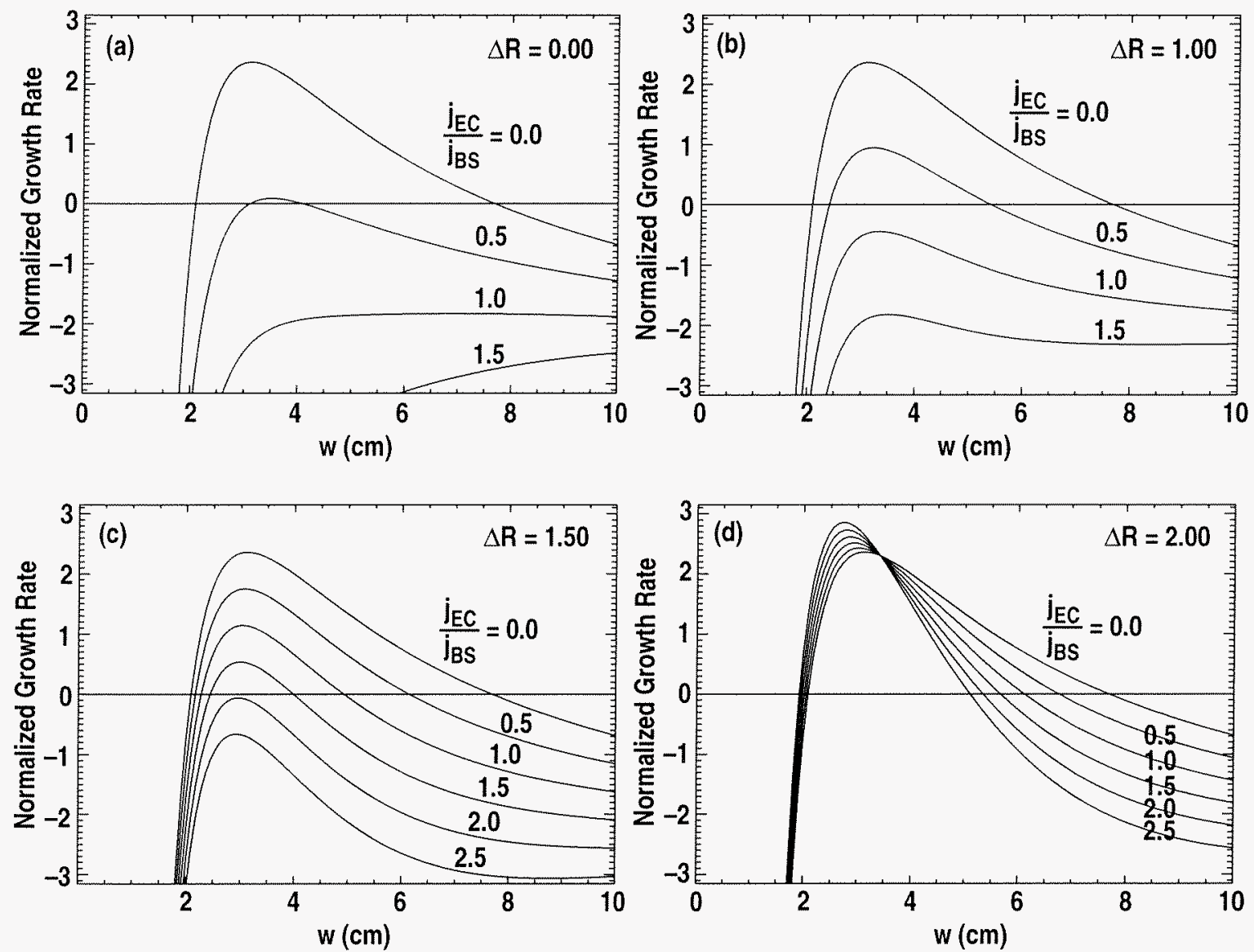

Fig. 1. (a) Normalized growth rate $\left(\tau_{R} / r\right) d w / d t$ of the $m=3 / n=2$ island as a function of the island full width $w$ for different levels of driven current density jEC normalized by the bootstrap current density $\mathrm{j}_{\mathrm{BS}}$, for the case of perfect alignment of the driven current with the center of the island and no modulation of the ECCD. (b) Same as (a), but the center of the ECCD is shifted by $1.0 \mathrm{~cm}$ from the location of the island. (c) Same as (b) but with a $1.5 \mathrm{~cm}$ shift. (d) Same as (b) but with a cm shift. The growth rate calculation uses parameters typical of the DIII-D discharges used in this study: $\Delta^{\prime} r=-3.2, w_{\text {pol }}=1.8 \mathrm{~cm}, \mathrm{j}_{\mathrm{BS}}=7.5 \mathrm{~A} / \mathrm{cm}^{2}, \mathrm{j}_{\mathrm{q}}=92 \mathrm{~A} / \mathrm{cm}^{2}, \mathrm{dq} / \mathrm{dr}=6.0 / \mathrm{m}$, and the calculated value $\delta_{\mathrm{EC}}=2.9 \mathrm{~cm}$.

Figure 1(a) also illustrates why the NTM is so suitable to feedback stabilization in these experiments: it is strongly stable in the limit of small island size, and growth takes place only after a minimum island size has been generated through some other mechanism like a sawtooth or an edge localized mode (ELM). This implies that the NTM control system does not need to address mode amplitudes at the limit of detection, and in theory the stabilizing power may be turned off between triggers after the mode has been reduced to an amplitude below the threshold size. This has not been demonstrated in practice yet.

The function $\mathrm{K}_{1}$ in Eq. (1) introduces the effectiveness of the ECCD, which may be reduced by inaccuracy in the location of the applied ECCD and the finite width of the current drive profile. This effect has been studied by Perkins [11] for the case of continuous current drive and for current drive modulated in phase with the island. For continuous current drive, as applied in these experiments, the effectiveness function $\mathrm{K}_{1}$ may be plotted as shown in Fig. 2 . 

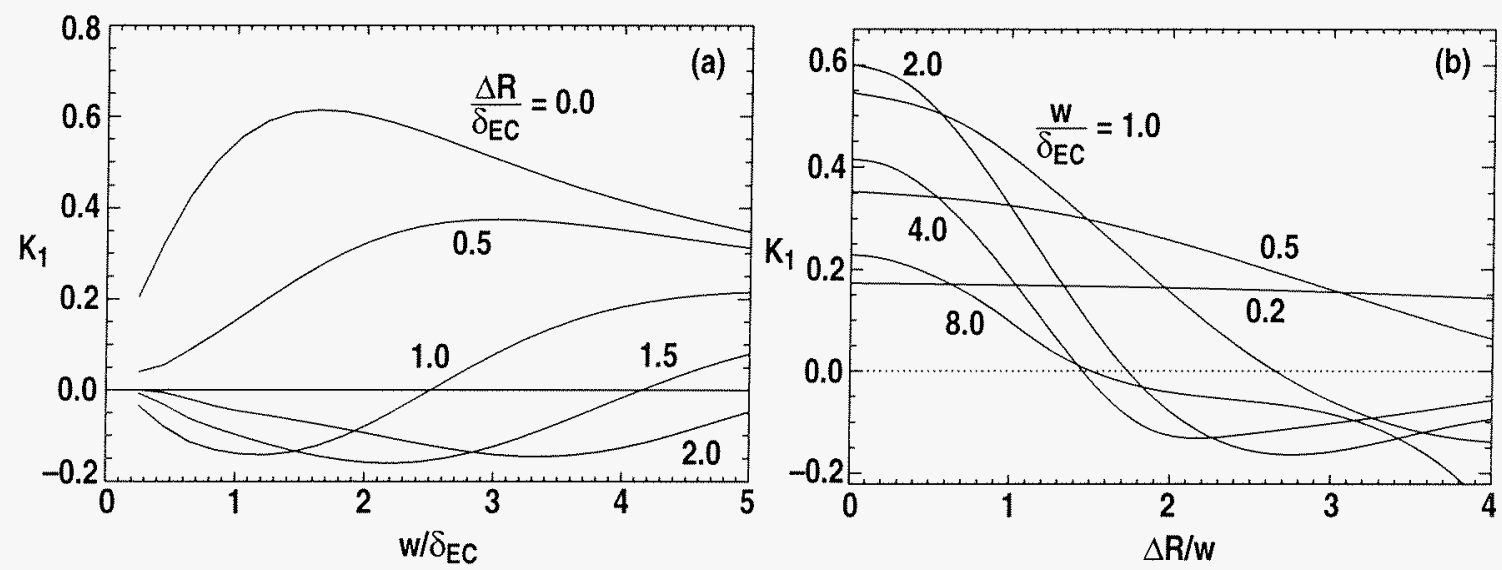

Fig. 2. Effectiveness $K_{1}$ of current drive in stabilizing an NTM. (a) $K_{1}$ plotted as a function of $w / \delta_{\mathrm{EC}}$, where $w$ is the island full width and $\delta_{\mathrm{EC}}$, is the FWHM of the driven current, for values of $\Delta \mathrm{R} / \delta_{\mathrm{EC}}$, (b) $\mathrm{K}_{1}$ plotted as a function of $\Delta \mathrm{R} / \mathrm{w}$ for values of $w / \delta_{\mathrm{EC}}$.

These curves show the strong sensitivity to $\Delta \mathrm{R}$ when $\mathrm{w} / \delta_{\mathrm{EC}} \sim 1$. Under this condition, the ECCD is destabilizing $\left(\mathrm{K}_{1}<0\right)$ for small $\mathrm{w} / \delta_{\mathrm{EC}}$ due to the large fraction of the driven current which lies outside the island.

The strong sensitivity of the stability condition to the displacement $\Delta \mathrm{R}$ can be illustrated by plotting the growth rate versus island size for different values of $\Delta R$ and $j E C / j B S$ as shown in Figs. 1(b-d). For a displacement of $1 \mathrm{~cm}$ [Fig. 1(b)] the required current density to fully stabilize the mode rises to about 0.8 jBs. For $1.5 \mathrm{~cm}$ displacement, Fig. 1(c) shows that jEC of 2 jBS is required to stabilize the mode. For $2.0 \mathrm{~cm}$ displacement, ECCD cannot stabilize the mode, as shown in Fig. 1(d). This seems reasonable since the displacement is larger than the island halfwidth at the island size of maximum growth rate, so much of the driven current lies outside the island where it has a mildly growth-inducing effect rather than a stabilizing effect. These curves show that accurate placement of the driven current is key to stabilization of the mode.

An additional complication is that the current drive applied at the location of the island will modify the equilibrium current profile, changing the classical stability quantity $\Delta^{\prime}$ in Eq. (1) [12]. In general, the effect is to flatten the current profile and improve the stability. The theory for dealing with both current drive within an island and modification of the equilibrium current profile has not been worked out. This effect is not considered in this paper, but it may be significant. 


\section{STABILIZATION OF THE 3/2 NTM BY ECCD}

Stabilization experiments on DIII-D were carried out using the $110 \mathrm{GHz}$ ECH system [13], using four gyrotrons with power incident on the plasma of about $0.55 \mathrm{MW}$ each. The EC power is directed at the plasma using steerable antennas. The arrangement is shown in Fig. 3. The ECCD is applied near the $q=3 / 2$ surface at the inboard midplane in order to minimize electron trapping effects which reduce the efficiency of the ECCD. In Fig. 3, the trajectories of 30 rays which are used to simulate the Gaussian beam from a single antenna are shown. The beam dispersion half angle at half power is 1.7 degrees.

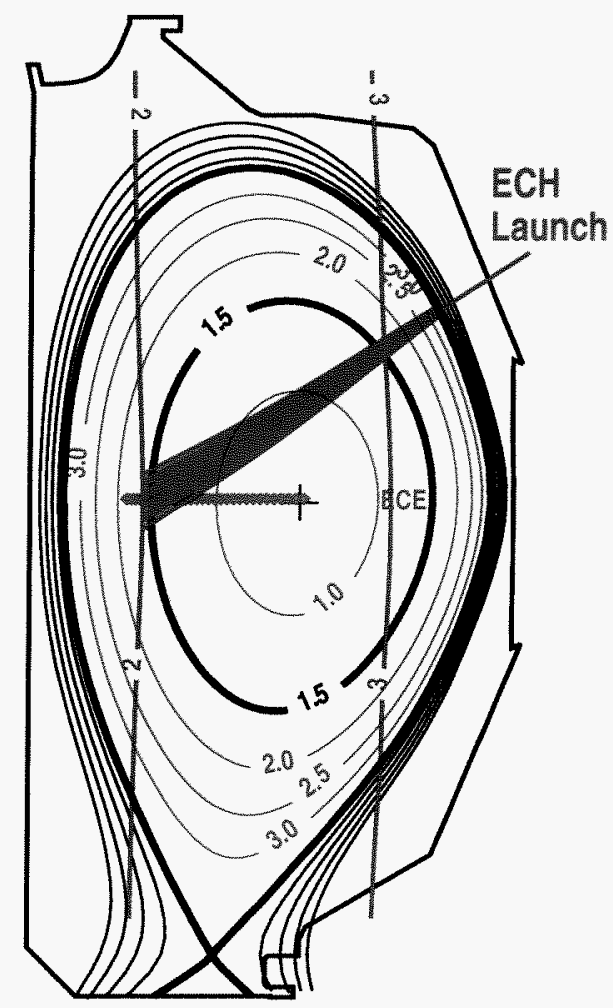

Fig. 3. Plasma equilibrium, with second and third harmonic electron cyclotron resonances superposed. Also shown is a bundle of 30 rays of equal power simulating the Gaussian beam propagation from a single ECH antenna. The toroidal field is $1.57 \mathrm{~T}$, the plasma current is $1.2 \mathrm{MA}$, the central electron temperature and density are $2.9 \mathrm{keV}$ and $5 \_10^{19} \mathrm{~m}^{-3}$.

The optimum EC launch conditions were determined for the target equilibrium by running the TORAY-GA ECH ray-tracing code for an array of poloidal and toroidal launch angles for the $\mathrm{EC}$ ray bundle, to find where $\mathrm{jEC}_{\mathrm{EC}}$ is maximum (rather than $\mathrm{I}_{\mathrm{EC}}$ being maximum) at the normalized minor radius where the value of the safety factor is $3 / 2$. The controlled parameters in this study are the poloidal steering angle (that is, the angle between a vertical and the ray bundle) 
and the $n_{\|}=c k_{\|} / \omega$ at the location where the EC power attenuation rate is maximum. These calculations are shown in Fig. 4, which shows the peak current density and the normalized minor radius $\rho$ of maximum absorption rate for a relevant range of poloidal angle and $n_{\|}$for the equilibrium shown in Fig. 3.

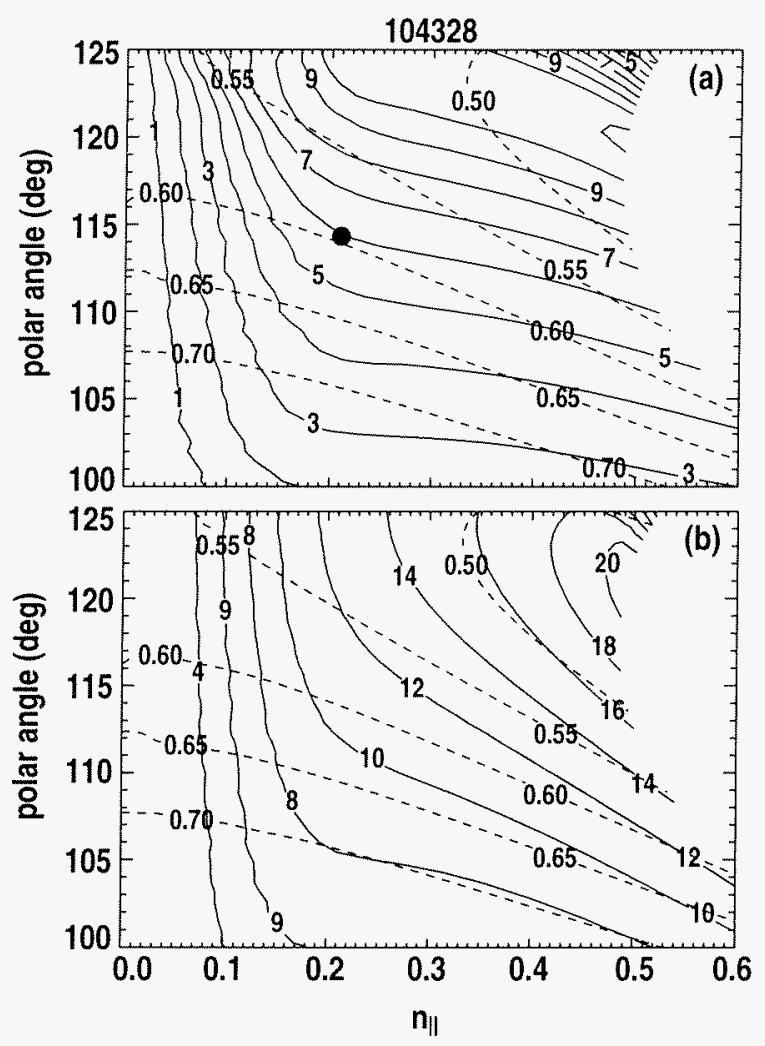

Fig. 4. Calculations with the TORAY-GA ray tracing code showing the peak ECCD current density (solid lines, $\mathrm{A} / \mathrm{cm}^{2}$ ) and the normalized minor radius (dashed lines) as a function of the poloidal launch angle of the ECH antenna and the local $n_{\|}$at the peak of the absorption, for the equilibrium and geometry of Fig. 3. The poloidal angle is the angle between the ray bundle and the vertical, and the sign convention for $\mathrm{n}_{\|}$is that $\mathrm{n}_{\|}>0$ drives co-current. (b) Same calculations as (a), but now the total driven current (kA/MW) is contoured instead of current density. The $q=3 / 2$ surface is near $\rho=0.6$, and the dot in (a) shows the parameters used. The plasma local density is $3.9 \times 10^{19} \mathrm{~m}^{-3}$, the local electron temperature is $1.5 \mathrm{keV}$, and the central toroidal field is $1.57 \mathrm{~T}$.

For this case, the $q=3 / 2$ surface is very near $p=0.6$. Figure 4 (a) shows that the $\mathrm{n}_{\|}$for optimum current density is near 0.2. The calculations in Fig. 4(b) show that larger $n_{\|}$may be expected to drive substantially larger total current, but due to the greater Doppler broadening the peak current density decreases. At smaller launch angles the current drive efficiency decreases too much to compensate for decreased Doppler width, and the peak current density also decreases. For this experiment each antenna was set to launch power only 9 degrees from radial in order to provide the n\| near 0.2 .

As discussed in the previous section, the precise location of the ECCD is critical. In order to find the optimum location experimentally, the co-ECCD power was first applied continuously 
while the magnetic field was slowly ramped down. Since the location of the ECCD is nearly fixed relative to the resonance, which in turn is dependent primarily on the magnitude of the toroidal field, this process sweeps the ECCD across the plasma, while the NTM is fixed to the $q=3 / 2$ surface which moves much less as the toroidal field decreases. The size of the NTM is followed by determining the amplitude of the $n=2$ component of the magnetic fluctuations (Mirnov signal) at the vacuum vessel wall through Fourier analysis of a toroidal array of magnetic pickup loops. A typical case is shown in Fig. 5.

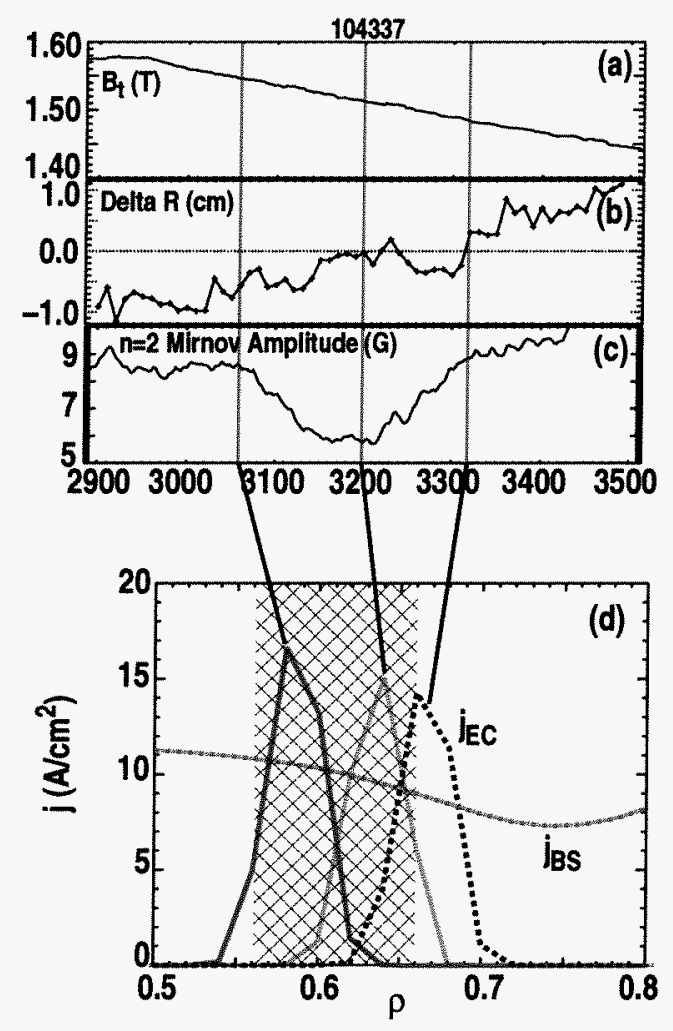

Fig. 5. (a) Toroidal magnetic field, (b) $\Delta R=R_{3 / 2}-R_{E C}$, (c) $n=2$ Mirnov amplitude as a function of time during a ramp-down of the toroidal field. (d) Calculated ECCD for 1.1 MW incident ECH power or the times corresponding to the bars in (a) through (c), for the discharge illustrated in Fig. 3. The shaded region corresponds to the width of the island prior to the ECCD.

The radial separation $\triangle R$ of the resonant surface from the location of the ECCD is shown in Fig. 5(b). The dip in the $n=2$ amplitude in Fig. 5(c) corresponds to the maximum efficacy of the ECCD. By running the TORAY-GA code for the times just before the dip, at the minimum of the dip, and just after the dip, the expected profile of $\mathrm{jEC}_{\mathrm{EC}}$ can be calculated for each case, as shown in Fig. 5(d). This figure shows that the three cases correspond to the minimum distinguishable separation of the current profiles; that is, the space between the peaks is about the same as the width of the peaks. Also shown in Fig. 5(d) is the full width of the island as determined from measurements of ECE fluctuations at the frequency of the $n=2$ magnetic fluctuation, before the ECCD is applied. The alignment of the ECCD with the magnetic island is clearly very sensitive experimentally, in agreement with theory. The bootstrap current density calculated by the 
ONETWO transport code is also shown in Fig. 5(d). The peak of the ECCD exceeds the bootstrap current density by $30 \%$ in this case. According to the calculation described in the previous section and in Fig. 1, this exceeds the level needed for full stabilization of the mode if the radial alignment is perfect. However, the sweep may be too fast for the full effect of the ECCD to be realized. This calculation of ECCD neglects absorption of EC power where the wave crosses the third electron cyclotron harmonic (shown in Fig. 3). If the power lost at the third harmonic is significant, then the driven current will be smaller in Fig. 5(d).

Since alignment of the applied current drive with the location of the island is a central element of the theory, the location of the absorbed EC power was tested by the method of modulating the EC power at $100 \mathrm{~Hz}$ and finding the location of the $100 \mathrm{~Hz}$ response on the ECE signals [14]. This location can then be compared with the location of the mode using the ECE response at the frequency of the mode, $12.5 \mathrm{kHz}$ [15]. Use of the same ECE channels in both measurements eliminates uncertainty in mapping the heating location to the mode location. Figure 6 shows the good alignment of the location of ECH power deposition with the $\mathrm{T}_{\mathrm{e}}$ fluctuations due to the magnetic island for the condition of the middle case of Fig. 5(b).
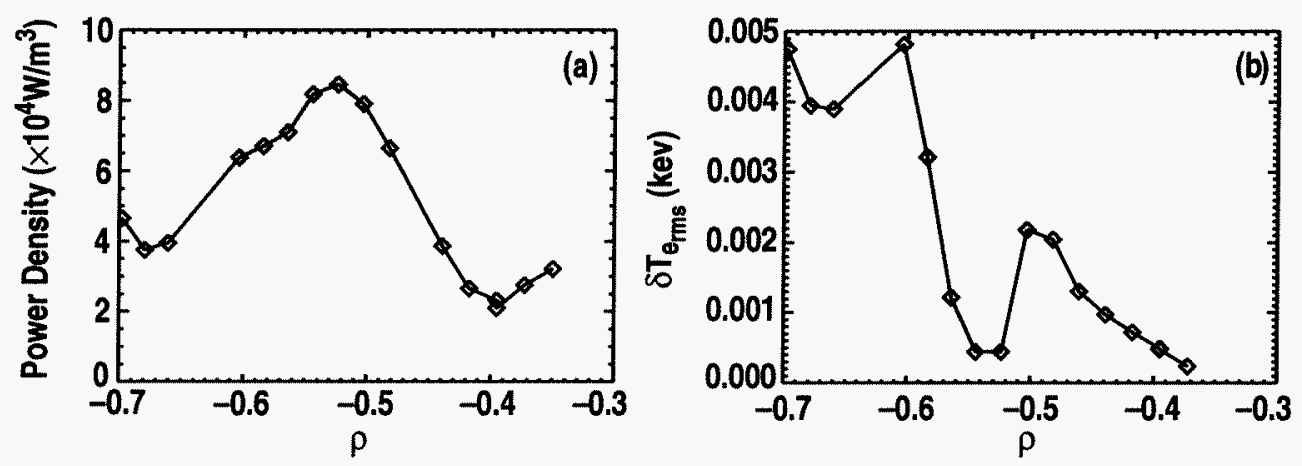

Fig. 6. (a) Power density inferred from ECE measurements during ECH modulated at $100 \mathrm{~Hz}$ versus normalized minor radius, for the conditions of Fig. 3. (b) RMS fluctuation in $\mathrm{T}_{\mathrm{e}}$ (keV) measured by ECE at the frequency of the NTM, $12.5 \mathrm{kHz}$, vs normalized radius. Negative $\rho$ indicates that the measurements are on the high field side of the plasma center. Note that the profile of power density in (a) is broadened substantially by transport.

When the ECCD is placed in the correct location corresponding to the maximum decrease of the amplitude in Fig. 5, an ECH power of 1.1 MW is sufficient to drive the NTM island size to zero. Figure 7 shows the application of ECCD in a discharge with $6 \mathrm{MW}$ of neutral beam heating and starting with a fully saturated 3/2 NTM present. The effect of the ECCD is to decrease the mode amplitude, and as this happens the normalized beta, $\beta_{\mathrm{N}}$, rises by $30 \%$ as shown in Fig. 7(c), where an increase of less than $10 \%$ can be attributed to additional heating power added by the ECH system. Hence, confinement is clearly improved by the suppression of the mode, also shown in Fig. 7(c). Figure 7(b) shows that as the $n=2$ mode decays the amplitude of an $n=1$ mode grows. This $n=1$ mode is the sawtooth precursor, and clearly the sawteeth become more vigorous when the $n=2$ mode has disappeared. Confinement nevertheless improves, as compared 
to the H89P scaling. Throughout this process, sawteeth and ELMs occur continuously as shown in Fig. 7(d).

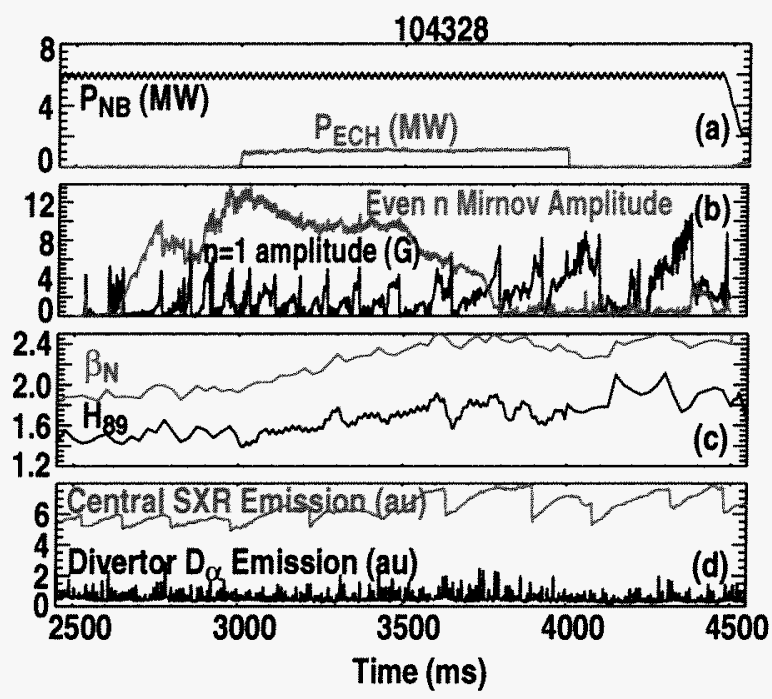

Fig. 7. Time traces for a discharge in which the ECCD causes a complete stabilization of the $\mathrm{m}=3 / \mathrm{n}=2$ neoclassical tearing mode. (a) Neutral injection and $\mathrm{ECH}$ power, (b) Mirnov amplitude for $m=3 / n=2$ and $m=2 / n=1$ modes, (c) $\beta_{N}$ and confinement factor $H$ relative to the ITER-89P scaling, and (d) central soft X-ray emission and $\mathrm{D}_{\alpha}$ light emission from the divertor. The equilibrium for this discharge is illustrated in Fig. 3.

The detailed behavior of the mode amplitude after the ECCD is applied can be used to study the physics of the mode suppression. Figure 7(b) shows that the mode amplitude shrinks rapidly at first and then gradually more slowly, until an island size is reached where the decay becomes fast and the mode decreases rapidly into the noise. This knee in the curve may be associated with the growth rate of the mode becoming negative and large; that is, $w<3.5 \mathrm{~cm}$ for the curve of Fig. 1(a) with $\mathrm{j}_{\mathrm{EC}} / \mathrm{j}_{\mathrm{BS}}=0.5$. After the $\mathrm{ECH}$ pulse is terminated, the figure shows that the mode growth does not resume, presumably because the $n=2$ amplitude does not reach the magnitude of the threshold needed for growth in the top curve of Fig. 1(a). 



\section{INCREASED PRESSURE LIMIT WHEN 3/2 NTM IS STABILIZED}

The stabilization of the $3 / 2$ NTM has a very practical benefit: the plasma pressure can be increased to well above the previous beta limit. Such a case is shown in Fig. 8. In this discharge the high power phase of the neutral beam heating is started at $1500 \mathrm{~ms}$, and $\beta_{\mathrm{N}}$ rises rapidly to 2.5 in response [Fig 8(c)]. This $\beta_{\mathrm{N}}$ is large enough that the $3 / 2 \mathrm{NTM}$ is destabilized at about $1900 \mathrm{~ms}$, and as this mode grows the $\beta_{\mathrm{N}}$ decreases to 1.7 due to an enhancement of transport caused by the island. At $3000 \mathrm{~ms}$ the power from four gyrotrons, $2.2 \mathrm{MW}$, is added to provide ECCD at the predetermined optimum location on the island center. The NTM amplitude immediately begins to decrease and, as in Fig. 7, it decreases very rapidly to near zero after the island shrinks below a threshold size.

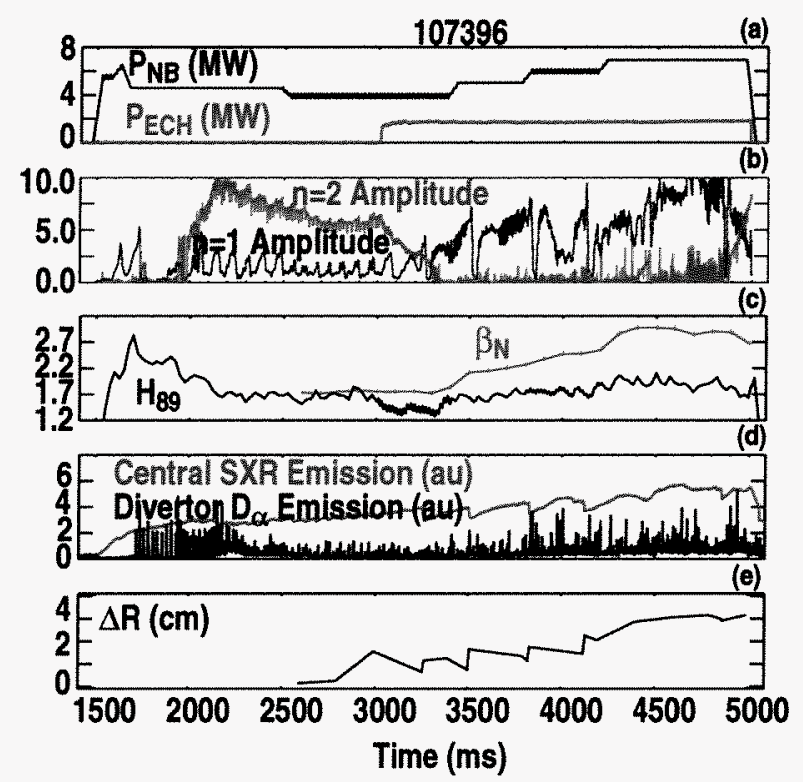

Fig. 8. Discharge in which the NBI power was raised following suppression of the $3 / 2$ NTM by ECCD. (a) Neutral injection and ECH power, (b) Mirnov amplitude for $m=3 / n=2$ and $m=2 / n=1$ modes, (c) $\beta_{\mathrm{N}}$ and confinement factor $\mathrm{H}$ relative to the ITER-89P scaling, (d) central soft $\mathrm{x}$-ray emission and $D_{\alpha}$ light emission from the divertor, and (e) $\Delta R=R_{3 / 2}-R_{E C}$, the radial displacement of the $q=3 / 2$ surface at the inboard midplane from the location of the ECCD. The equilibrium and geometry are similar to that shown in Fig. 3.

After the amplitude of the 3/2 NTM falls to zero the neutral injection power is increased in preprogrammed steps. The $\beta_{\mathrm{N}}$ increases in response to the greater heating power. At the highest NBI power used, $7 \mathrm{MW}$, the $\beta_{\mathrm{N}}$ has increased to 3.0 , or nearly double the $\beta_{\mathrm{N}}$ when the $3 / 2 \mathrm{NTM}$ is saturated, and $20 \%$ above the $\beta_{\mathrm{N}}$ at which the mode originally started to grow. Since plasma reactivity is proportional to $\beta^{2}$, these are important advances in the plasma stability. 
It can be seen from Fig. 8 that the plasma energy confinement is smaller at $4000 \mathrm{~ms}$, when the ECH is stabilizing the NTM, than at $1750 \mathrm{~ms}$ before the mode starts. At least part of this loss of confinement may be attributed to the fishbone instabilities which arise after the NTM is stabilized, as well as to the decrease in confinement associated with the higher input power. The fishbones are identified as creating the $n=1$ signal in Fig. 8(b), and the periodic modulation of this signal is due to sawteeth, which also grow in size and decrease in frequency. Presumably the lower level of MHD activity when the NTM is absent improves the confinement of fast ions from the neutral beam injection. Then the density of fast ions can rise to a large enough level to trigger the fishbones, and fishbones are known to be efficient in transporting fast ions toward the plasma edge. 


\section{MAINTAINING THE OPTIMUM LOCATION OF THE ECCD}

It is important that the location of the ECCD retain good alignment with the location of the island. In the discharge of Fig. 8, the $3 / 2$ NTM restrikes at $4850 \mathrm{~ms}$ at the crash of a sawtooth, even though the ECCD is still being applied. Growth at this sawtooth crash and not at previous crashes is consistent with Eq. (1), since the ECCD is no longer at the optimum position. When the mode is initially stabilized, the plasma center begins moving to larger major radius due to the increased Shafranov shift associated with the increased beta, while the ECCD location which is anchored primarily to the toroidal field does not. To illustrate this point, the movement of the location of the $q=3 / 2$ surface at the inboard midplane, as determined from numerical reconstructions of the equilibrium, from its original position just before the ECH is shown in Fig. 8(e). At the time of the mode growth the displacement has reached $2.5 \mathrm{~cm}$. The FWHM $\delta_{\mathrm{EC}}$ of the current drive profile as calculated by the TORAY-GA code is $2.9 \mathrm{~cm}$, and allowing for reasonable relative misalignment of the antennas and enhancement of the $\mathrm{EC}$ wave dispersion due to density fluctuations the width is still less than $4 \mathrm{~cm}$. From Fig. 2 with $\Delta R=0$, it may be seen that the effectiveness of the ECCD in suppressing the NTM at $4850 \mathrm{~ms}$ is reduced to less than $20 \%$ of its initial value. Hence, the mode may grow when a sawtooth generates a trigger island of sufficient size.

Recognizing that changes to the plasma parameters while the mode is not present can reduce the ability of the ECCD to suppress a mode when a trigger occurs, several strategies have been developed to address this. First, an automated "search and suppress" mechanism has been set up using the digital plasma control system (PCS). In this approach, ECCD is applied at some preset location. If mode growth occurs (as measured by real time analysis of the signals from an array of Mirnov coils), the assumption is that the ECCD is not in the optimum location. Through the PCS, the location of the ECCD is shifted by a small amount relative to the island and maintained for a brief period, typically $100 \mathrm{~ms}$ in these experiments. If the mode shrinks but doesn't vanish, another shift in the same direction is produced until the mode either stops shrinking or vanishes. If the mode doesn't shrink, the location is shifted further through a predetermined number of steps, and if no shrinkage occurs the direction is reversed. When the mode vanishes, the location of the ECCD is held fixed. If the initial location is near the optimum location, this process can find the optimum and maintain it through sufficiently small or slow movements of the plasma. Knowledge about which way to move is not required.

Several methods of changing the relative location of the ECCD are possible, but the applicability of the methods depends on the geometry. If the ECCD is at the inboard midplane, as in Fig. 3, the most effective approach is to have the plasma control system request a shift of the plasma major radius to move the island or of the toroidal magnetic field to move the ECCD. A prefixed step corresponding to a change $\Delta \mathrm{R}$ of the relative ECCD location by about $1 \mathrm{~cm}$ is used. 
Both of these approaches have been successfully tested on DIII-D [8]. Figure 9 shows the trajectories of two example discharges. In one the major radius was shifted and in the other the toroidal field was changed in a search for the optimum location. The $3 / 2$ mode in each case starts with the amplitude saturated, and the plasma control system carries out a series of shift/dwell steps in major radius of about $1 \mathrm{~cm}$ each, which culminate in the full suppression of the mode. Discharges in which the initial guess for the direction was wrong have also been successfully stabilized by this process.

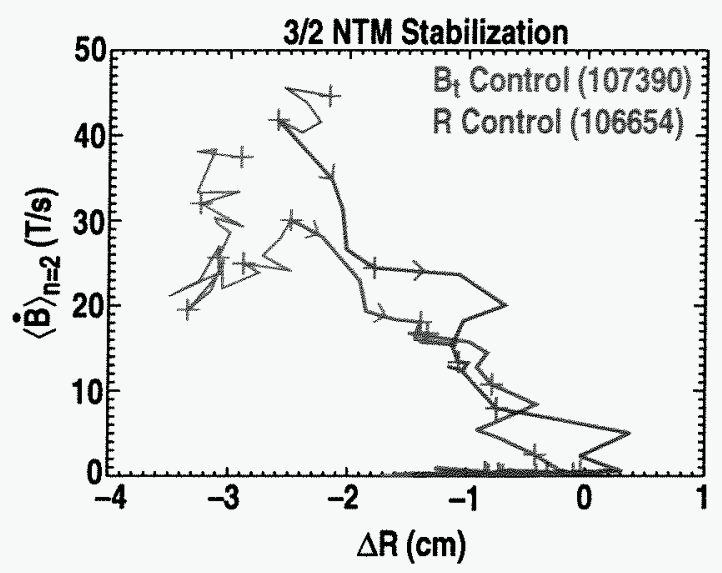

Fig. 9. Trajectory of the amplitude of the $n=2$ magnetic perturbation measured at the vacuum vessel wall (related to the size of the $3 / 2$ magnetic island) versus the displacement of the $q=3 / 2$ surface from the surface where the ECCD is maximum, measured at the inboard midplane. For discharge 107390 (green curve) the toroidal field is modified by the plasma control system to search for the optimum location for suppression of the 3/2 NTM, and for discharge 106654 (red curve) the plasma is shifted radially by the PCS. In both cases the initial location was not optimum and complete suppression was obtained after 1 or 2 steps. The ECCD is applied where the curves are thicker, and the crosses are placed on the traces every $100 \mathrm{~ms}$. The arrowheads indicate the progression in time.

If the poloidal location of the ECCD on a flux surface lies above the plasma center instead of at the inboard midplane, neither of the two approaches described above are effective in shifting the relative location of the ECCD. For this situation, a plasma shift in the vertical direction is more effective, or the launch angle in the vertical plane of the EC waves may be changed. These techniques will be tested in the future. In any case, the "blind search" used in this work may be improved by obtaining real time estimates of the magnitude and direction of the optimum step size. This is being carried out on DIII-D through use of real time equilibrium reconstructions as part of the PCS. 


\section{CONCLUSIONS}

Localized ECCD applied at the optimum location has proved to be a reliable and robust means of fully suppressing the $3 / 2$ NTM, in validation of theoretical predictions which originated before experiments were attempted [4,5]. When the NTM is stabilized the plasma beta rises more than expected from the additional heating power of the $\mathrm{ECH}$, and when further beam heating is added the beta can be raised to as high as $60 \%$ above the beta when the saturated mode is present. Several means of keeping the ECCD at the appropriate location through a seeking process of small shifts of the plasma location or the toroidal field have been used successfully. 